\title{
Spin Precession Mapping at Ferromagnetic Resonance via Nuclear Resonant Scattering of Synchrotron Radiation
}

\author{
Lars Bocklage, ${ }^{1,2,}$ Christian Swoboda,${ }^{3,2}$ Kai Schlage, ${ }^{1}$ Hans-Christian Wille, ${ }^{1}$ Liudmila Dzemiantsova, ${ }^{1,2}$ \\ Saša Bajt, ${ }^{1}$ Guido Meier, ${ }^{4,3}$ and Ralf Röhlsberger ${ }^{1,2}$ \\ ${ }^{1}$ Deutsches Elektronen-Synchrotron DESY, Notkestraße 85, 22607 Hamburg, Germany \\ ${ }^{2}$ The Hamburg Centre for Ultrafast Imaging, Luruper Chaussee 149, 22761 Hamburg, Germany \\ ${ }^{3}$ Institut für Nanostruktur und Festkörperphysik, Universität Hamburg, Jungiusstrasse 11, 20355 Hamburg, Germany \\ ${ }^{4}$ Max-Planck Institute for the Structure and Dynamics of Matter, Luruper Chaussee 149, 22761 Hamburg, Germany
}

(Received 25 September 2014; published 6 April 2015)

\begin{abstract}
We probe the spin dynamics in a thin magnetic film at ferromagnetic resonance by nuclear resonant scattering of synchrotron radiation at the $14.4 \mathrm{keV}$ resonance of ${ }^{57} \mathrm{Fe}$. The precession of the magnetization leads to an apparent reduction of the magnetic hyperfine field acting at the ${ }^{57} \mathrm{Fe}$ nuclei. The spin dynamics is described in a stochastic relaxation model adapted to the ferromagnetic resonance theory by Smit and Beljers to model the decay of the excited nuclear state. From the fits of the measured data, the shape of the precession cone of the spins is determined. Our results open a new perspective to determine magnetization dynamics in layered structures with very high depth resolution by employing ultrathin isotopic probe layers.
\end{abstract}

DOI: 10.1103/PhysRevLett.114.147601

PACS numbers: 76.50.+g, 75.25.-j, 76.80.+y, 78.70.Ck

Spin waves, collective excitations of the magnetization, are key features of magnetic materials as they determine switching times and energy losses during magnetization reversal. They play a crucial role for new concepts of logical operations, spin-torque oscillators, or signal processing, and constitute the basis of the emerging field of magnonics [1]. Spin waves are often probed by inelastic scattering techniques like Raman scattering, Brillouin light scattering, or inelastic neutron scattering that rely on analysis of the energy transfer to the scattered particles. We show that resonant spin dynamics can be probed by coherent elastic scattering, namely, nuclear resonant scattering of synchrotron radiation (NRS) [2,3]. Since NRS is an x-ray technique, it opens the possibility to combine it with diffraction methods to obtain very high spatial resolution [4] down to atomic length scales [2]. Moreover, as NRS probes the nuclear decay of an excited Mössbauer isotope, it allows one to employ ultrathin isotopic probe layers to achieve sub-nm depth resolution [5].

The application of conventional Mössbauer spectroscopy as well as NRS has given indirect access to thermally excited spin waves in ferromagnets via the temperature dependence of the magnetic hyperfine field under the assumption that it exhibits the same temperature dependence as the magnetization in these materials [6-8]. In contrast to a broad thermally excited spin wave spectrum, we induce a single coherent mode by resonant excitation with radio frequency (rf) magnetic fields. This is one approach typically used in magnonic devices to obtain the desired functionality $[9,10]$.

The impact of $\mathrm{rf}$ magnetic fields on conventional Mössbauer spectroscopy has so far been studied at $\mathrm{MHz}$ frequencies that are well below ferromagnetic resonances.
It was shown that low-frequency spin waves in paramagnetic media can induce magnetic phase modulations of the nuclear states [11]. A collapse of the magnetic hyperfine field arises from a fast periodic switching of the magnetization $[12,13]$. The sideband effect originates from acoustic vibrations induced by magnetostriction [13,14]. Also, Rabi oscillations have been observed where the $\mathrm{rf}$ field directly couples to the nuclear transition [15]. Ferromagnetic or spin wave resonances at $\mathrm{GHz}$ frequencies have not been investigated with Mössbauer spectroscopy or NRS so far. This is the case to be studied here.

In this Letter we show that NRS measurements enable one to extract the trajectory of the spins during coherent precession at ferromagnetic resonance. The opening angle of the precession is an essential parameter for spintronics like, e.g., in spin pumping [16,17] or in the spin dynamo [18]. So far, the determination of the opening angle from experiments appeared to be challenging. Values for opening angles averaged over time and space have been determined by anisotropic magnetoresistance measurements under the assumption of circular magnetization trajectories [19,20], which is justified only in a few special cases like in spherical particles without crystalline anisotropy.

We perform NRS measurements on ferromagnetic thin films excited at ferromagnetic resonance. The influence of spin dynamics on the NRS signal is analyzed in a stochastic relaxation model [21,22] adapted to the ferromagnetic resonance theory of Smit and Beljers [23]. With this method the exact shape of the precession orbit is determined. This capability arises from the high sensitivity of NRS to the magnetization direction.

NRS in grazing-incidence geometry $[3,24]$ is performed at the Dynamics beam line P01 [25] of PETRA III at DESY 
(Hamburg, Germany) in 40 bunch mode with a bunch separation of $192 \mathrm{~ns}$ and a bunch duration of about $50 \mathrm{ps}$. The energy is tuned to the nuclear transition energy of $14.4125 \mathrm{keV}$ of the Mössbauer isotope ${ }^{57} \mathrm{Fe}$ that has a natural lifetime of $141 \mathrm{~ns}$. The bandwidth of the synchrotron radiation is reduced to $1 \mathrm{meV}$ by a high resolution monochromator. A Kirkpatrick-Baez multilayer mirror system focuses the beam to a cross section of about $10 \times 10 \mu \mathrm{m}^{2}$. The synchrotron pulse excites all six dipole-allowed nuclear transitions simultaneously. Their frequency differences manifest as quantum beats in the temporal evolution of the nuclear decay. The recorded NRS time spectra are fingerprints for the magnetic spin structure of the sample.

Samples are prepared on GaAs substrates. All layer geometries are defined by electron-beam lithography and lift-off processing. Electrical striplines are prepared by thermal evaporation of $7 \mathrm{~nm} \mathrm{Cr}, 118 \mathrm{~nm} \mathrm{Ag}$, and $20 \mathrm{~nm} \mathrm{Au}$. Hydrogen silsesquioxane with a thickness of $140 \mathrm{~nm}$ is used to electrically insulate the strip line from the ferromagnetic film and to provide a smooth surface. The $800 \times$ $800 \mu \mathrm{m}^{2}$ film is prepared by sputter deposition of $4 \mathrm{~nm} \mathrm{Cr}$, $18 \mathrm{~nm} \mathrm{Pd}$, and $13 \mathrm{~nm}$ isotopically enriched Permalloy $\left(\mathrm{Ni}_{81}\right.$ ${ }^{57} \mathrm{Fe}_{19}$ ) [26], and finally capped by $2 \mathrm{~nm} \mathrm{Pd}$. A scheme of the layer system is shown in Fig. 1(a). The stoichiometry of Permalloy is confirmed by energy-dispersive $\mathrm{x}$-ray spectroscopy. The sideband effect is negligible in Permalloy due to the low magnetostriction and the high frequencies [15]. Because the ferromagnetic resonance of the film is only effectively excited right above the $10 \mu \mathrm{m}$ wide stripline, any NRS signal from nonexcited parts of the magnetic film has to be blocked which is achieved by a highly x-ray absorbing bilayer of $7 \mathrm{~nm} \mathrm{Al}$ and $30 \mathrm{~nm}$ Au on top of the magnetic film. The sample is illuminated under grazing incidence at an angle $\varphi$ of $4.36 \mathrm{mrad}$, which corresponds to the critical angle for total reflection where the nuclear signal reaches its maximum [27]. The $10 \times$ $800 \mu \mathrm{m}^{2}$ large rf-excited part of the Permalloy film is completely illuminated by the microbeam at the given angle of incidence.
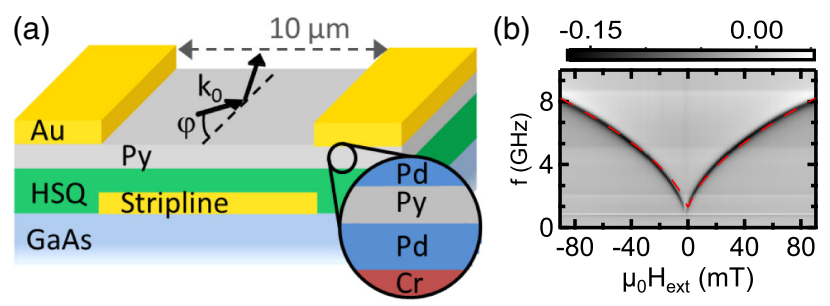

FIG. 1 (color online). (a) Schematic layer system of the sample (see text). The angle of incidence of the photon wave vector $k_{0}$ is $\varphi$. (b) Absorption spectrum of the Permalloy film depending on external field and excitation frequency. The grey scale gives the transmission in arbitrary units. Black indicates high absorption. Dashed red line is the fit to the Kittel formula.
External fields are applied in the plane of the film. The stripline is connected to a vector network analyzer that serves as a signal source for the high frequency excitation as well as detector for the transmitted signal. Figure 1(b) shows an electrical absorption spectrum [28] of the film where high absorption (black) indicates the excitation of the ferromagnetic resonance (Kittel mode) of the Permalloy film [29], a spin wave mode with zero wave vector that corresponds to a uniform precession of the magnetic moments. A fit with the Kittel formula yields a saturation magnetization of $M_{S}=666 \mathrm{kA} / \mathrm{m}$ and a damping parameter of $\alpha=0.012$.

The precise evaluation of the NRS time spectra requires the exact knowledge of the hyperfine field distribution in the sample. This can be obtained from the evaluation of NRS time spectra without rf magnetic field excitation at different in-plane angles $\phi$ between the incoming photon wave vector $k_{0}$ and an external field of $70 \mathrm{mT}$. Because of the low coercivity of the Permalloy film of less than $1 \mathrm{mT}$ [30], the magnetization $m$ and the external field are parallel. The NRS time spectra are shown in Fig. 2 together with fits via the program package conUss [31,32], from which a hyperfine field distribution of the Permalloy film centered around 27.6 $\mathrm{T}$ has been deduced, as shown in the inset of Fig. 2.

In the following, the Kittel mode is excited at different static fields applied parallel to the incoming beam. Time spectra for a constant external field of $5 \mathrm{mT}$ at different radio-frequency magnetic field amplitudes $h_{\mathrm{rf}}$ are shown in Fig. 3(a). The resonance frequency is $1.93 \mathrm{GHz}$ as determined from the electrical absorption measurements. The overall shape of the time spectra changes with increasing excitation field amplitudes. A shift of the extrema to later times with increasing dynamic field is

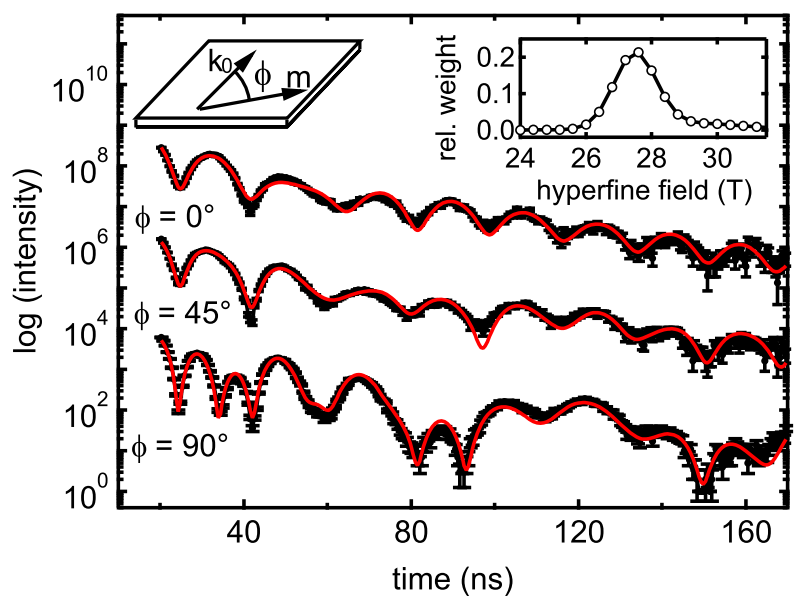

FIG. 2 (color online). NRS time spectra from the Permalloy film at different in-plane angles $\phi$ between the photon wave vector $k_{0}$ and the magnetization $m$. The red lines show fits using the theory of nuclear forward scattering [31,32]. Curves at $0^{\circ}$ and $45^{\circ}$ are offset for clarity. The deduced hyperfine field distribution of the Permalloy film is given in the inset. 
visualized in Fig. 3(b). In addition, a slightly faster decay of the time spectra is observed during ferromagnetic resonance. The shift of the extrema indicates that the effective magnitude of the hyperfine field has decreased with increasing dynamic field. It results in a reduction of the magnetic level splitting and a correspondingly larger period in the temporal beat pattern. The effect resembles the temperature dependence of the hyperfine field that originates from thermal excitation of spin waves [6-8]. However, here we excite only one coherent mode which allows one to determine dynamic magnetic properties under conditions of ferromagnetic resonances.

The hyperfine interaction energy $E_{\mathrm{hf}}=\left(m_{\mathrm{e}} g_{\mathrm{e}}-\right.$ $\left.m_{\mathrm{g}} g_{\mathrm{g}}\right) B_{\mathrm{hf}}=\hbar \omega_{\mathrm{hf}}$ determines the time scale for dynamic effects on the NRS signal $[33,34]$. Here, $m_{\mathrm{g}}$ and $m_{\mathrm{e}}$ are the magnetic quantum numbers and $g_{\mathrm{g}}$ and $g_{\mathrm{e}}$ are the $g$ factors of the nuclear ground and excited states, respectively. For average hyperfine fields of $27.6 \mathrm{~T}$ observed in Permalloy, the frequency $\omega_{\text {hf }} / 2 \pi$ is in the order of a few $10 \mathrm{MHz}$. For any temporal change of the hyperfine field much faster than $1 / \omega_{\mathrm{hf}}$, the nucleus cannot follow the hyperfine field and thus, it experiences an effective hyperfine field resulting from temporal averaging over the relatively long lifetime of the nuclear excited state. This situation corresponds to

(a)

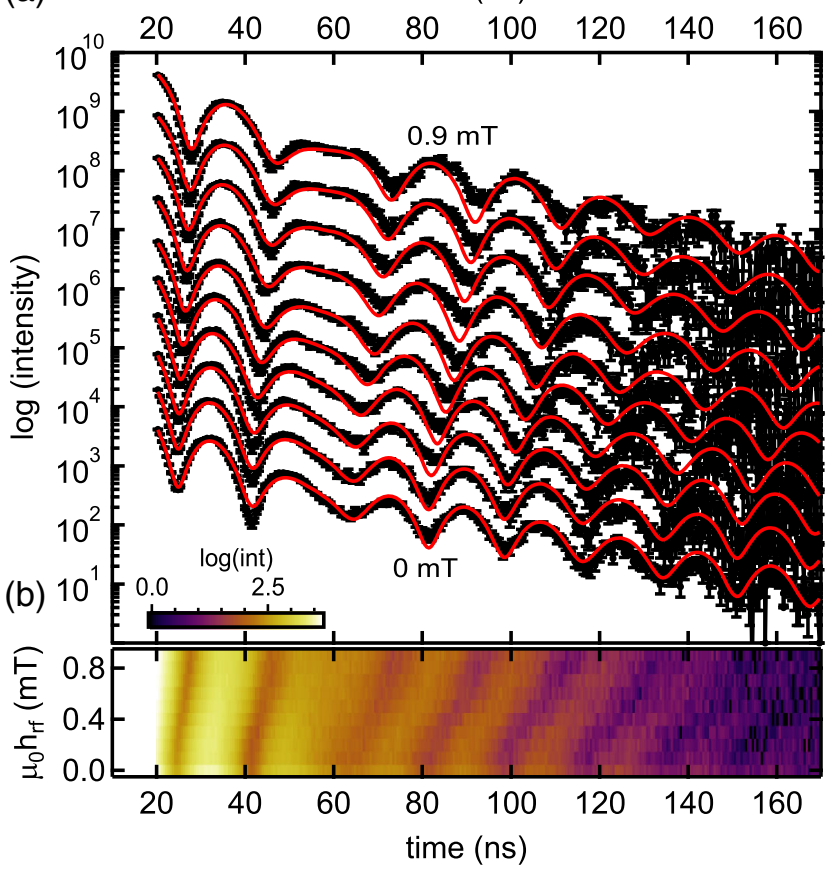

FIG. 3 (color online). (a) Time spectra of the Permalloy film $\left(\phi=0^{\circ}\right)$ at various rf excitation field amplitudes for a frequency of $1.93 \mathrm{GHz}$. The lowest time spectrum is not excited. The excitation field $\mu_{0} h_{\mathrm{rf}}$ increases up to $0.9 \mathrm{mT}$ in steps of $0.1 \mathrm{mT}$. Curves with excitation are offset for clarity. Red lines are fits with the stochastic model as explained in the text. (b) Logarithmic intensity map of the time spectra shown in (a) with varying excitation field $h_{\mathrm{rf}}$. the fast relaxation regime. As a consequence, a reduced effective hyperfine field is observed for a precession of the magnetization around the equilibrium direction. Moreover, the high sensitivity of NRS to the magnetization direction, as shown in Fig. 2, yields the capability to determine the precession orbit of the magnetization because its dynamic in-plane and out-of-plane components influence the time spectra.

For a quantitative evaluation of the influence of the spin wave on the time spectra, calculations within a stochastic relaxation model are performed [21,22], which is implemented in CONUSS to account for temporal variations of the hyperfine field. The stochastic model assumes discrete hyperfine field directions with transition rates $t_{\mathrm{nm}}$ between field directions $n$ and $m$. We model the spin precession by eight points on the precession cone as shown in Fig. 4(a). The transition matrix is chosen to allow transitions between neighboring points in a way that supports only one sense of rotation as realized in the experiment [27]. The transition rates equal the inverse period of the spin wave, meaning that every transition is performed once per cycle.

The field directions on the precession trajectory have to be modeled for the calculations in the stochastic model. In the thin film, the demagnetization field that leads to an elliptical precession has to be considered. We deduce the precession trajectory of the magnetization from the SmitBeljers formulation of the ferromagnetic resonance [23]. The Smit-Beljers formulation yields a set of coupled differential equations for the dynamic components $\delta \varphi$ and $\delta \vartheta$ of the azimuthal angle $\varphi$ and polar angle $\vartheta$ of the magnetization (see Fig. 4) [35]

$$
\begin{aligned}
-\gamma^{-1} M_{S} \sin \left(\vartheta_{0}\right) \delta \dot{\vartheta} & =F_{\varphi \vartheta} \delta \vartheta+F_{\varphi \varphi} \delta \varphi, \\
\gamma^{-1} M_{S} \sin \left(\vartheta_{0}\right) \delta \dot{\varphi} & =F_{\vartheta \vartheta} \delta \vartheta+F_{\vartheta \varphi} \delta \varphi,
\end{aligned}
$$

where $\gamma$ is the gyromagnetic ratio, $\vartheta_{0}$ is the equilibrium polar angle, and $F$ is the free energy density. The indices of $F$ indicate partial derivatives at equilibrium positions with respect to these variables. From Eq. (1) we derive the ratio of the dynamic angles at resonance

$$
\frac{\delta \varphi}{\delta \vartheta}=-i \sqrt{\frac{F_{\vartheta \vartheta}}{F_{\varphi \varphi}}},
$$

when the magnetization lies in the azimuthal film plane. Without crystalline anisotropy, the free energy density of the thin film is $F=-\mu_{0} M_{S} H_{\text {ext }} \sin \vartheta \cos \varphi+\frac{1}{2} \mu_{0} M_{S}^{2} \cos ^{2} \vartheta$, where $\varphi$ is the in-plane angle between magnetization and external field. At equilibrium the magnetization and external field are parallel $\left(\vartheta_{0}=90^{\circ}\right.$ and $\left.\varphi_{0}=0^{\circ}\right)$ and we get 


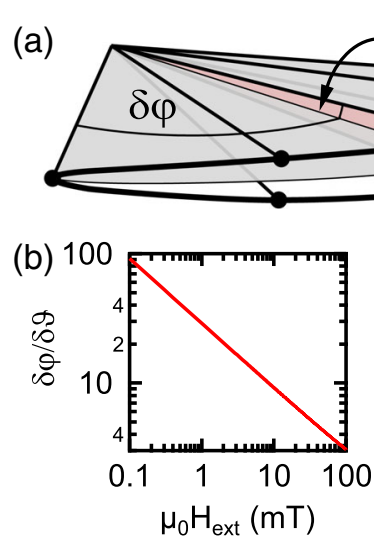

$\delta \vartheta$
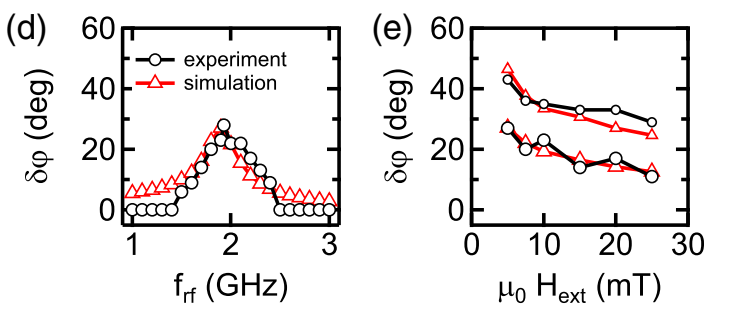

FIG. 4 (color online). (a) Scheme showing the discrete points on the magnetization precession cone used in the stochastic relaxation model. Gray indicates the film plane and red the outof-plane direction. $\delta \varphi$ and $\delta \theta$ are the dynamic in-plane and outof-plane angles, respectively. The black dots represent the magnetization directions with transition rates $t_{n m}$ used in the calculations of the NRS time spectra. (b) Ratio of the dynamic angles for the Permalloy film. (c)-(e) Maximum in-plane angle $\delta \varphi$ of the spin precession cone in dependence of the excitation field amplitude at an external field of $5 \mathrm{mT}$ with a resonance frequency of $1.93 \mathrm{GHz}$ (c), of the excitation frequency at a field amplitude of $0.4 \mathrm{mT}(\mathrm{d})$, and of the external field at the resonance frequency with $\mathrm{rf}$ magnetic field amplitudes of 0.4 and $0.8 \mathrm{mT}$ (smaller symbols) (e). Data deduced from the measurements (circles) and data from micromagnetic simulations (triangles) are shown. In (c) the dependence of the reduced hyperfine field on the rf magnetic field is shown with diamonds. Lines are guides to the eye.

$$
\left|\frac{\delta \varphi}{\delta \vartheta}\right|=\sqrt{\frac{H_{\mathrm{ext}}+M_{S}}{H_{\mathrm{ext}}}} .
$$

This ratio for the film, shown in Fig. 4(b), is inserted in the CONUSS calculations for the parametrization of the precession cone. For example, the experimental conditions presented in Fig. 3 with an external field of $5 \mathrm{mT}$ yield a ratio of 13.0 [see Fig. 4(b)]. The maximum dynamic inplane angle $\delta \varphi$ is the only free parameter in the fits of the time spectra under spin wave excitation.

Figures 4(c)-4(e) show the in-plane opening angle $\delta \varphi$ deduced from the time spectra. Figures 4(c) and 4(d) display data at an external field of $5 \mathrm{mT}$ where the resonance frequency is $1.93 \mathrm{GHz}$. For increasing $\mathrm{rf}$ excitation fields the increase of the opening angle is almost linear up to $0.4 \mathrm{mT}$. At higher rf fields the opening angles deviate from a linear dependence showing that the nonlinear regime is reached [Fig. 4(c)]. With an excitation field of $0.4 \mathrm{mT}$ the opening angles around resonance in Fig. 4(d) are obtained. Figure 4(e) shows the opening angle at resonance for different static external fields. For increasing fields, the opening angle is reduced.

The values of the extracted dynamic angles are compared to micromagnetic simulations. These simulations have been carried out with the program package MicroMagnum [36] with a film thickness of $12.9 \mathrm{~nm}$ using a cell size of $5 \times 5 \times 4.3 \mathrm{~nm}^{3}$, a saturation magnetization of $666 \mathrm{kA} / \mathrm{m}$, a Gilbert damping [37] of 0.012 , and periodic boundary conditions for the $x$ and $y$ directions. The external field has been slightly adjusted by a few percent in the simulation to fit the experimentally observed resonance frequency. The opening angles calculated from the simulations are also shown in Figs. 4(c)-4(e). The calculated angles agree well with the values obtained from the NRS spectra. The excellent agreement of the measured and simulated data for the thin film demonstrates how powerful NRS is to sense spin dynamics. Especially for samples that are nanoscaled in all three dimensions, where theoretical modeling might get difficult, NRS could provide distinct advantages in determining dynamic properties.

The measurements indeed allow one to distinguish between different assumed trajectories. The simplest model is a circular precession cone. This model fits the data quite reasonably because the average hyperfine field is the projection on the equilibrium direction of the magnetization. The slightly faster decay of the NRS time spectra due to the dynamics is reproduced as well. However, the dynamic in-plane and out-of plane components $\delta \varphi$ and $\delta \vartheta$ of the magnetization precession change for the circular trajectory compared to the elliptical one. This change generates differences in the time spectra fit quality and yields poorer fits with up to $9 \%$ larger least squares. However, we can simply deduce the effective hyperfine field from the cosine of the circular cone opening angle. The reduced hyperfine field $B_{\mathrm{hf}}\left(h_{\mathrm{rf}}\right) / B_{\mathrm{hf}}(0)$ obtained in this manner is shown in Fig. 4(c). At an excitation field of $0.9 \mathrm{mT}$ a reduction of $10 \%$ is observed. For small opening angles the hyperfine field reduction becomes less significant. Because the effective hyperfine field scales with the average of the cosine of the dynamic angles, the change of the hyperfine field is very small. For the smallest deduced in-plane angle of $6^{\circ}$, the out-of-plane angle is $0.46^{\circ}$ and we get hyperfine field reductions of $0.55 \%$ and $0.003 \%$, respectively. However, these small reductions are sufficient to induce changes in the NRS time spectra compared to the static case.

In summary we have shown that the magnetic hyperfine field is drastically modified at ferromagnetic resonance. Nuclear resonant scattering measurements enable one to deduce the precession orbit of the magnetization in thin film systems. The overall good agreement of the 
micromagnetic simulations and the evaluated data demonstrate the feasibility to study spin dynamics with high accuracy via NRS. The method's isotopic sensitivity can be employed to study depth profiles and interface effects [5] related to spin dynamics by placing isotopic probe layers in selected depths of the sample. A combination of grazingincidence diffraction and time-resolved NRS will enable three-dimensional mapping of spin waves confined in nanostructures.

We thank U. Merkt and T. Matsuyama for persistent encouragement and fruitful discussions, M. Volkmann and A. Berg for excellent technical support, A. Aquila for the software code for KB mirror calculations, A. Rothkirch for help during the data processing, C. Adolff for support during lithography, J. Major for production of a sputter target, and D. Schumacher as well as T. Guryeva for sputter deposition. Financial support of the Deutsche Forschungsgemeinschaft via excellence cluster "The Hamburg Centre for Ultrafast Imaging: Structure, Dynamics, and Control of Matter on the Atomic Scale," via Sonderforschungsbereich 668 and via Graduiertenkolleg 1286 is gratefully acknowledged.

*lars.bocklage@desy.de

[1] V. V. Kruglyak, S. O. Demokritov, and D. Grundler, J. Phys. D 43, 264001 (2010).

[2] E. Gerdau, R. Rüffer, H. Winkler, W. Tolksdorf, C. P. Klages, and J. P. Hannon, Phys. Rev. Lett. 54, 835 (1985).

[3] R. Röhlsberger, Nuclear Condensed Matter Physics with Synchrotron Radiation, Springer Tracts in Modern Physics, Vol. 208 (Springer, Berlin, 2005).

[4] K. Schlage, S. Couet, S. V. Roth, U. Vainio, R. Rüffer, M. M. A. Kashem, P. Müller-Buschbaum, and R. Röhlsberger, New J. Phys. 14, 043007 (2012).

[5] R. Röhlsberger, H. Thomas, K. Schlage, E. Burkel, O. Leupold, and R. Rüffer, Phys. Rev. Lett. 89, 237201 (2002).

[6] M. Eibschütz and M. E. Lines, Phys. Rev. B 7, 4907 (1973).

[7] C. L. Chien and R. Hasegawa, Phys. Rev. B 16, 2115 (1977).

[8] V. Senz, R. Röhlsberger, J. Bansmann, O. Leopold, and K.-H. Meiwes-Broer, New J. Phys. 5, 47 (2003).

[9] S. O. Demokritov, V. E. Demidov, O. Dzyapko, G. A. Melkov, A. A. Serga, B. Hillebrands, and A. N. Slavin, Nature (London) 443, 430 (2006).

[10] M. P. Kostylev, A. A. Serga, T. Schneider, B. Leven, and B. Hillebrands, Appl. Phys. Lett. 87, 153501 (2005).
[11] T. W. Sinor, P. W. Reittinger, and C. B. Collins, Phys. Rev. Lett. 62, 2547 (1989)

[12] L. Pfeiffer, J. Appl. Phys. 42, 1725 (1971).

[13] M. Kopcewicz, Struct. Chem. 2, 313 (1991).

[14] N. D. Heiman, L. Pfeiffer, and J. C. Walker, Phys. Rev. Lett. 21, 93 (1968).

[15] I. Tittonen, M. Lippmaa, E. Ikonen, J. Lindén, and T. Katila, Phys. Rev. Lett. 69, 2815 (1992).

[16] Y. Tserkovnyak, A. Brataas, G. E. W. Bauer, and B. I. Halperin, Rev. Mod. Phys. 77, 1375 (2005).

[17] N. Kuhlmann, C. Swoboda, A. Vogel, T. Matsuyama, and G. Meier, Phys. Rev. B 87, 104409 (2013).

[18] Y. S. Gui, N. Mecking, X. Zhou, G. Williams, and C.-M. Hu, Phys. Rev. Lett. 98, 107602 (2007).

[19] M. V. Costache, S. M. Watts, M. Sladkov, C. H. van der Wal, and B. J. van Wees, Appl. Phys. Lett. 89, 232115 (2006).

[20] N. Kuhlmann, A. Vogel, and G. Meier, Phys. Rev. B 85, 014410 (2012).

[21] M. Blume, Phys. Rev. 174, 351 (1968).

[22] M. J. Clauser and M. Blume, Phys. Rev. B 3, 583 (1971).

[23] J. Smit and H. G. Beljers, Philips Res. Rep. 10, 113 (1955).

[24] R. Röhlsberger, J. Bansmann, V. Senz, K. L. Jonas, A. Bettac, K. H. Meiwes-Broer, and O. Leupold, Phys. Rev. B 67, 245412 (2003).

[25] http://photon-science.desy.de/facilities/petra_iii/beamlines/ p01_dynamics/index_eng.html.

[26] The iron is enriched to $95 \%$ in ${ }^{57} \mathrm{Fe}$.

[27] See Supplemental Material at http://link.aps.org/ supplemental/10.1103/PhysRevLett.114.147601 for nuclear reflectivity and for details on data evaluation.

[28] L. Bocklage, S. Motl-Ziegler, J. Topp, T. Matsuyama, and G. Meier, J. Phys. Condens. Matter 26, 266003 (2014).

[29] C. Kittel, Rev. Mod. Phys. 21, 541 (1949).

[30] T. Kamionka, M. Martens, A. Drews, B. Krüger, O. Albrecht, and G. Meier, Phys. Rev. B 83, 224424 (2011).

[31] W. Sturhahn and E. Gerdau, Phys. Rev. B 49, 9285 (1994).

[32] W. Sturhahn, Hyperfine Interact. 125, 149 (2000).

[33] M. Blume and J. A. Tjon, Phys. Rev. 165, 446 (1968).

[34] O. Leupold and H. Winkler, Hyperfine Interact. 123/124, 571 (1999).

[35] G. Skrotskii and L. Kurbatov, in Ferromagnetic Resonance, edited by S. Vonsovskii (Pergamon Press, Oxford, 1966) pp. 12-77.

[36] MicroMagnum Fast Physical Simulator for Computations on CPU and Graphics Processing Unit, http://micromagnum .informatik.uni-hamburg.de/.

[37] T. L. Gilbert, IEEE Trans. Magn. 40, 3443 (2004). 